\title{
Video Article \\ Using the Threat Probability Task to Assess Anxiety and Fear During Uncertain and Certain Threat
}

\author{
Daniel E. Bradford ${ }^{* 1}$, Katherine P. Magruder ${ }^{* 1}$, Rachel A. Korhumel ${ }^{1}$, John J. Curtin ${ }^{1}$ \\ ${ }^{1}$ Department of Psychology, University of Wisconsin-Madison \\ *These authors contributed equally
}

Correspondence to: John J. Curtin at jjcurtin@wisc.edu

URL: http://www.jove.com/video/51905

DOI: doi:10.3791/51905

Keywords: Behavior, Issue 91, Startle; electromyography; shock; addiction; uncertainty; fear; anxiety; humans; psychophysiology; translational

Date Published: 9/12/2014

Citation: Bradford, D.E., Magruder, K.P., Korhumel, R.A., Curtin, J.J. Using the Threat Probability Task to Assess Anxiety and Fear During Uncertain and Certain Threat. J. Vis. Exp. (91), e51905, doi:10.3791/51905 (2014).

\section{Abstract}

Fear of certain threat and anxiety about uncertain threat are distinct emotions with unique behavioral, cognitive-attentional, and neuroanatomical components. Both anxiety and fear can be studied in the laboratory by measuring the potentiation of the startle reflex. The startle reflex is a defensive reflex that is potentiated when an organism is threatened and the need for defense is high. The startle reflex is assessed via electromyography (EMG) in the orbicularis oculi muscle elicited by brief, intense, bursts of acoustic white noise (i.e., "startle probes"). Startle potentiation is calculated as the increase in startle response magnitude during presentation of sets of visual threat cues that signal delivery of mild electric shock relative to sets of matched cues that signal the absence of shock (no-threat cues). In the Threat Probability Task, fear is measured via startle potentiation to high probability (100\% cue-contingent shock; certain) threat cues whereas anxiety is measured via startle potentiation to low probability ( $20 \%$ cue-contingent shock; uncertain) threat cues. Measurement of startle potentiation during the Threat Probability Task provides an objective and easily implemented alternative to assessment of negative affect via self-report or other methods (e.g., neuroimaging) that may be inappropriate or impractical for some researchers. Startle potentiation has been studied rigorously in both animals (e.g., rodents, non-human primates) and humans which facilitates animal-to-human translational research. Startle potentiation during certain and uncertain threat provides an objective measure of negative affective and distinct emotional states (fear, anxiety) to use in research on psychopathology, substance use/abuse and broadly in affective science. As such, it has been used extensively by clinical scientists interested in psychopathology etiology and by affective scientists interested in individual differences in emotion.

\section{Video Link}

The video component of this article can be found at http://www.jove.com/video/51905/

\section{Introduction}

The overall goal of the Threat Probability Task is to experimentally disentangle the expression of anxiety in response to low probability (i.e., uncertain) threats from fear in response to high probability (i.e., certain) threats. Uncertainty occurs when some aspect of a threat is poorly defined. While anxiety can be described in numerous ways, exacerbated responses to low probability or otherwise uncertain negative events is a distinguishing clinical symptom in anxiety disorders ${ }^{1,2}$. Furthermore, increased anxiety related physiological responding during uncertain threat of shock versus fear related physiological responding during certain threat of shock in laboratory tasks may provide a physiological marker for anxiety disorders ${ }^{3}$. Dampening of anxiety to uncertain threats specifically may be a critical component of the stress response dampening properties of drugs such as alcohol ${ }^{4-7}$. Increased anxiety during uncertain threat may mark a neuroadaptation in the brain's stress circuitry following chronic drug use $e^{4,8}$. Thus, the Threat Probability Task provides an objective measure of negative affective and distinct emotional states (anxiety, fear) to use in research on psychopathology, substance use/abuse and affective science. As such, it can be a powerful tool for use by clinical and affective scientists interested in psychopathology etiology and individual differences in emotion.

\section{Traditional methods used to study emotions in humans}

Affective scientists have used numerous measures and paradigms to study human emotion ${ }^{9}$ but most of these do not provide the necessary precision found in the Threat Probability Task to parse anxiety from other negative emotions such as fear. For example, self-report is commonly used but it may suffer from demand characteristics and other forms of response bias. Participants may not be able to accurately distinguish between anxiety and fear, and the connection of their report to underlying neurobiological mechanisms is distal at best. Furthermore, self-report must often be conducted retrospectively since the process of introspection and report might otherwise alter participants' experience of the affective stimuli. Of course, retrospective report suffers from memory interference and degradation. Psychophysiologists often measure emotions during an affect manipulation that involves presentation of emotionally evocative pictures ${ }^{10}$. This picture viewing task is well validated, is less affected by the shortcomings of self-report, and has resulted in many important insights regarding individual differences in affective response and their contribution to psychopathology ${ }^{11,12}$. However, only broad negative affect is measured during this picture viewing task which does not allow for the study of distinct negative emotions such as anxiety and fear which can be measured with the Threat Probability Task. Affective neuroscientists frequently measure functional magnetic resonance imaging (fMRI) during tasks that elicit negative affect but these approaches 
may be too costly for many researchers. Furthermore, the spatial and temporal resolutions of fMRI methods are currently limited, making it difficult for fMRI to disentangle the neurological structures believed to be associated with anxiety versus other emotions. More importantly, a welldefined fMRI index of any type of negative affect has yet to be established.

\section{Translational research with animals using the startle response}

The Threat Probability Task is modeled after basic research with animals that provided the first example of the precision needed to disentangle anxiety from fear. Neuroscientists have used carefully controlled lesion studies with rodents to model anxiety and fear using differential responses to uncertain and certain cued threat of electric shock. This work has elucidated important differences in anxiety related responses to low probability, ambiguously defined, distal or otherwise uncertain shock versus fear related responses to highly probable, clearly defined, imminent certain shock ${ }^{13}$. Uncertain threats elicit freezing and hyper vigilance in animals, whereas certain threats elicit active avoidance, defensive attack, or both ${ }^{14}$. Imminent, certain threats focus attention on the threat itself, whereas distal, temporally uncertain threats encourage distributed attention to the overall environment ${ }^{15-17}$. Response to temporally uncertain threats appears to be sustained, whereas response to certain threats is phasic and time-locked to the threat ${ }^{13}$. In related work, lesion studies have shown that response to uncertain threats are selectively mediated by corticotrophin-releasing factor and norepinephrine pathways through the lateral divisions of the central nucleus of the amygdala and the bed nucleus of the stria terminalis ${ }^{18}$. Much of this work uses potentiation of the acoustic startle response as a primary dependent measure ${ }^{13}$, which is the same dependent measure used in the Threat Probability Task. The neurobiological substrates of the startle response circuit have been extensively studied with the discovery of clear connections to the brain structures active in responses to uncertain and certain threats ${ }^{19,20}$. The startle response can be assessed in numerous species which provides a powerful translational tool to study emotions. The startle response in humans occurs reflexively in response to a sudden and intense auditory stimulus. Startle is most often measured in humans by the placement of electromyography (EMG) electrodes on the orbicularis oculi (lid closing) muscle of the eye. Startle related EMG activity is potentiated when an organism is presented with a threatening stimulus such as an impending electric shock relative to non-threatening stimuli ${ }^{19}$.

\section{The No-shock, Predictable-shock, Unpredictable-shock (NPU) task and threat uncertainty}

The Threat Probability Task was inspired by Grillon and colleagues when these researchers introduced the use of startle potentiation to study anxiety and fear in humans with the No-shock, Predictable-shock, Unpredictable-shock (NPU) task ${ }^{21}$. In the Predictable condition of the NPU task, shocks are 100 percent cue-contingent and occur at a consistent, known time (end of brief cue presentation). In the Unpredictable condition of the NPU task, shocks are fully unpredictable. Patients with posttraumatic stress and panic disorders exhibit selectively increased startle potentiation during unpredictable but not predictable shock in the NPU task ${ }^{22,23}$. In other work, medications prescribed to treat anxiety have a greater effect on startle potentiation during unpredictable shock than during predictable shock in the NPU task ${ }^{24}$. In research on the anxiolytic effects of alcohol, Moberg and Curtin ${ }^{4}$ used the NPU task to demonstrate that a moderate dose of alcohol selectively reduces startle potentiation during threat of unpredictable but not predictable shock. Uncertainty is multifaceted and shocks in the unpredictable condition of the NPU task are uncertain in regards to both IF they are to occur (probability uncertainty) and WHEN they occur (temporal uncertainty). Many theories suggest that the WHEN dimension of uncertainty is critical in producing anxiety ${ }^{19}$. However, data from Curtin et al. ${ }^{5}$ suggests a common mechanism for the elicitation of anxiety across various types of uncertainty. The Threat Probability task described here manipulates uncertainty about IF a shock will occur while holding all other dimensions of uncertainty constant thus making clear what aspect of uncertainty is responsible for the effects the task presents. Tasks that use startle potentiation to cued threat are flexible and can also be modified by affective scientists to manipulate uncertainty about WHERE the shocks are going to occur ${ }^{25}$ and HOW BAD they will be ${ }^{7,26}$. Of all of these tasks, the Threat Probability Task is one of the easiest to interpret due to its focus on one dimension of uncertainty and most straightforward to implement due to its inclusion of only two threat uncertainty variants (low probability and high probability shock).

\section{The Threat Probability Task}

In the Threat Probability Task, the participant is seated approximately $1.5 \mathrm{~m}$ from a cathode ray tube (CRT) monitor. Threat cues are displayed on the monitor for $5 \mathrm{sec}$ each with a variable duration ITI (range $=15-20 \mathrm{sec}$ ). Threat cues are divided into sets of two shock threat conditions and one no-threat condition (see Figure 1). In both threat conditions shocks of 200 msec duration are delivered at 4.5 sec into cue presentation times to the participant's fingers. In the $100 \%$ threat probability condition, shocks are delivered during presentation of every cue. In $20 \%$ threat probability condition, shocks are delivered during presentation of 1 out of every 5 cues. The participant sees two sets (15 cues total) of each threat probability condition. The participant also sees two neutral sets of cues that signal no threat (no-threat cues; 15 cues total). Text displayed on the monitor informs the participant of the next set type. A label for the set type is displayed during the entire set in the upper left corner of the monitor. Different color cues are used for each condition to facilitate awareness of each set for the participant. Throughout the task, the stimulus presentation program presents the participant with acoustic startle probes in the form of 50 msec bursts of $102 \mathrm{~dB}$ white noise with near instantaneous rise time delivered through headphones. Acoustic startle probes are delivered at $4 \mathrm{sec}$ into the presentation of a subset of the cues. Additional probes are delivered at $13 \mathrm{sec}$ and $15 \mathrm{sec}$ post cue offset during the ITIs to decrease the predictability of the probes. Before any presentation of visual stimuli, the task begins with the delivery of 3 acoustic startle probes to habituate the startle response immediately before main task measurement. Researchers balance the serial position of the acoustic startle probes across conditions within subjects in order to control for habituation and sensitization effects ${ }^{27,28}$. For an example of one fully counterbalanced series of trials for the Threat Probability Task see Supplementary Material.

The Threat Probability task has been used to demonstrate that low probability (uncertain) shock alone is sufficient to elicit anxiety and allow assessment of the anxiolytic effects of alcohol ${ }^{6}$. Preliminary research with dependent marijuana users suggests the Threat Probability Task can also be used to assess the effects of drug withdrawal ${ }^{29}$. Thus, the Threat Probability Task provides an easily implemented alternative to more expensive and less precise methods for the objective measure of distinct negative emotional states (e.g., anxiety and fear) for research on psychopathology, substance use/abuse, and broad affective science. 


\section{Protocol}

The local ethics committee has approved the following procedure and all participants who have taken part in this procedure have given informed consent. For additional detail of psychophysiological measurement and stimulus presentation please see ${ }^{30,27}$.

\section{Electromyography (EMG) Recording Preparation}

1. Ask the participant to wash their face thoroughly with soap, paying particular attention to the target sensor locations, which are located below one eye and in the middle of the participant's forehead (see Figure 2).

2. Seat the participant in a comfortable upright chair in the experimental chamber.

3. Prepare the participant's skin for EMG measurement.

1. Clean the target sensor locations with an alcohol pad.

2. Clean the same locations with a gritty exfoliate gel using a small gauze pad to further remove dirt or dead skin cells that can impede measurement of the electromyographic activity.

4. Prepare and attach EMG electrodes.

1. Fill all silver-silver chloride $(\mathrm{Ag}-\mathrm{AgCl})$ sensor cups with conductive gel using a syringe and blunt needle.

2. Attach a large (e.g., $8 \mathrm{~mm}) \mathrm{Ag}-\mathrm{AgCl}$ sensor to the center of the participant's forehead using an adhesive collar.

3. Attach two additional small (e.g., $4 \mathrm{~mm}$ ) Ag-AgCl sensors below the participant's eye using adhesive collars. Place the first of these small sensors in line with the pupil at forward gaze and the second sensor $1-2 \mathrm{~cm}$ lateral to the first (Figure 2; see also ${ }^{27}$ ). Do not allow the adhesive collars to overlap as this may increase movement artifact. Prevent gel overflow to avoid forming a gel bridge between the two sensors below the eye as this will cause current to flow via the bridge and impair measurement of EMG activity.

5. Start the EMG acquisition software on the physiology computer and ask the participant to blink a few times to verify that the EMG response is being recorded properly and that eye blinks can be observed on the display of the data collection software (see Figure 3A for an example of EMG activity associated with a blink).

6. Check the impedance for each sensor.

NOTE: Many laboratories require impedances below $10 \mathrm{k} \Omega$ (or more conservatively, $5 \mathrm{k} \Omega$ ) but actual tolerable thresholds for measured impedance levels depend on many variables such as experimental design, amplifier design, and practical constraints with respect to time needed to reduce impedances and the participant population. Regardless, high impedances increase susceptibility of the EMG signal to electrical artifact, which may be problematic (60 Hz noise; see Figure 3B).

7. Place the headphones on the participant's head.

\section{Baseline Measurement of General Startle Reactivity}

NOTE: This assessment also serves to further habituate the startle response prior to the three habituation probes delivered just before task start $^{31}$. Including general startle reactivity as a covariate in statistical analysis of startle potentiation increases statistical power to detect within and between participant effects. General startle reactivity may also reflect an interesting individual difference measure ${ }^{12,32}$.

1. Ask the participant to get comfortable before the start of the baseline task and to remain as still as possible throughout the task with their feet flat on the floor. Participant movement may introduce artifact into the EMG signal (see Figure 3C).

2. Remind the participant that they can discontinue their participation at any point during the experiment. Monitor the participant using video and audio feed from the experimental chamber during both the baseline assessment and main task.

3. Save the EMG signal with acquisition software on the physiology computer and start the stimulus presentation software on the stimulus control computer.

4. Present the participant with a series of colored squares that will be used in the main task but have not yet been paired with electric shock. Present startle probes during a subset of these cues and the interval between the cues. Timing parameters for cue duration, interval between cues, and startle probes should match parameters from the main task. Reliable measurement of general startle reactivity requires the presentation of at least 4 probes. This baseline task takes approximately 5 min to complete.

5. Average together the participant's peak EMG startle response to each startle probe in the baseline procedure to produce one value which will serve as this participant's general startle reactivity (see steps 6.1-6.6 for how to process the EMG data). Include general startle reactivity as an additive or interactive covariate in statistical models involving startle potentiation (see step 6.8).

\section{Shock Tolerance Threshold Assessment}

1. Affix two shock electrodes with standard medical tape to the participant's hand (e.g., distal phalanges of index and ring fingers of the hand $)^{33-35}$.

2. Present the participant with a series of increasingly intense electric shocks. After each shock is administered, ask the participant to rate how aversive they found the shock on a 100-point scale. Ask them to use a rating of 0 if they cannot feel a shock at all, a rating of 50 for the first level of shock that they consider to be uncomfortable, and a rating of 100 for the highest level of shock that they can tolerate.

3. Instruct the participant that it is important to accurately report the highest shock they can tolerate. The participant should not be informed that their report will impact the actual shocks they receive as this may lead to bias in their report.

4. Stop the shock tolerance assessment once the participant rates a shock as 100 . Record the shock level and administer shocks at this level in the Threat Probability Task to control for individual differences in shock sensitivity. 
NOTE: Electric shocks are administered to each participant's subjective maximum shock tolerance threshold. However, lower intensity shocks are also used ${ }^{21}$. Regardless, it is important that the shock intensity selected is sufficient to elicit a robust negative affective response and associated startle potentiation from all participants.

\section{The Threat Probability Task}

1. Provide the participant with a cover story that encourages attention throughout the task

NOTE: Some participants may find it difficult to maintain attention throughout the Threat Probability Task. An example of a cover story that researchers can tell participants in order to encourage attention in this task is to tell the participant that the researchers are interested in measuring the participant's ability to pay attention over time during a simple, repetitive visual task similar to the task required of air traffic controllers.

2. Provide the participant with general task information and specific cue-shock contingencies for each condition.

1. Instruct the participant that the task lasts approximately $20 \mathrm{~min}$.

2. Instruct the participant that the task includes cues that last 5 sec each separated by $15-20$ sec on average.

3. Inform the participant that the cues are organized into sets with each set lasting 2-3 min each.

4. Instruct the participant that there are three types of sets, $20 \%$ shock sets, $100 \%$ shock sets and No shock sets.

5. Instruct the participant that they will receive shocks at the end of approximately 1 out of every 5 cues in $20 \%$ shock sets and 5 out of every 5 cues in $100 \%$ shock sets.

6. Assure the participant that they will receive no shocks at any time during No shock sets or during the time between the presentations of the cues (ITI) in any of the sets.

7. Allow the participant to ask questions about the task at the end of the instructions. Following this, quiz the participant to make sure they completely understand the shock contingencies. Remind the participant that they can discontinue their participation at any point during the experiment.

3. Save the EMG signal with acquisition software on the physiology computer and start stimulus presentation software on the stimulus control computer that will control task stimuli.

4. Carefully monitor the participant for voluntary movements, closing of eyes, or excessive discomfort.

\section{Post-experiment}

1. After the cued threat task, administer a questionnaire to the participant to verify that the threat contingencies were well understood during the task. Ask the participant to rate how anxious or fearful they were when they saw each threat cue on a 5 point rating scale from 1 (not at all anxious/fearful) to 5 (extremely anxious/fearful).

NOTE: Results from Bradford et al. ${ }^{7,25}$ using two separate threat uncertainty tasks have shown a pattern of results in self-reported anxiety which closely matched that of startle potentiation.

2. Debrief the participant, compensate them for their time, and dismiss them.

3. Clean and disinfect all sensors.

\section{Data Processing, Reduction, and Analysis}

NOTE: Researchers can accomplish data processing and reduction with various software packages. EEGLAB ${ }^{36}$ is a free, open source toolbox for analysis of psychophysiological data within Matlab ${ }^{37}$. For a template EEGLAB script of data processing and reduction steps please see the supplementary material. Data processing and reduction follow published guidelines ${ }^{27}$. For a display of a few seconds of the unprocessed (raw) continuous EMG signal surrounding one startle probe, see Figure 4A.

1. Apply a forward-backward high pass filter $\left(4^{\text {th }}\right.$ order $28 \mathrm{~Hz}$ Butterworth filter) to the raw continuous EMG (see Figure 4 A,B).

2. Rectify the filtered continuous EMG (see Figure 4C).

3. Smooth the rectified EMG signal using a forward-backward $4^{\text {th }}$ order $30 \mathrm{~Hz}$ Butterworth low pass filter (see Figure 4D).

4. Epoch the smoothed continuous signal, retaining -50 to $250 \mathrm{msec}$ surrounding the acoustic startle probe onset and "Baseline correct" the epoched signal by subtracting the mean of the pre-probe baseline (-50 to $0 \mathrm{msec}$ ) from the entire epoched signal (see Figure 4E).

5. Score startle response from each epoch as maximum response between 20 and 100 msec post-probe onset (see Figure 4F).

6. Reject trials with excessive artifact (e.g., excessive deflections in the pre-probe baseline; see Figure 5). NOTE: Signals that contain greater than $40 \mu \mathrm{V}$ deflections in the pre-probe baseline may be identified as artifact.

7. Average startle response for epochs within each task condition (no-shock, $20 \%$ shock, $100 \%$ shock) (see Figure 6A).

1. Calculate startle potentiation for uncertain shock as the difference between mean startle response to startle probes during $20 \%$ shock cues vs. no-shock cues (see Figure 6B). NOTE: Startle response to ITI probes during the $20 \%$ condition can also be measured to study effects of anticipation and sustained startle potentiation relevant to some conceptualizations of anxiety ${ }^{6,21}$.

2. Calculate startle potentiation for certain shock as the difference between mean startle response to startle probes during $100 \%$ shock cues vs. no-shock cues (see Figure 6B).

8. Analyze startle potentiation using a General Linear Model with repeated measures on task condition and general startle reactivity (calculated in step 2.5) as an additive or interactive covariate ${ }^{32}$.

\section{Representative Results}

The Threat Probability Task produces robust startle potentiation during both $100 \%$ (certain) probability and $20 \%$ (uncertain) probability threat cues (see Figure 6B). Previous results using this task show startle potentiation during the uncertain (20\%) threat condition to be significantly increased above startle potentiation during high probability (100\%) (certain) threat condition. Acute administration of a moderate does of alcohol 
(target blood alcohol concentration of $0.08 \%$ ) produces selectively greater reduction in startle potentiation during $20 \%$ (uncertain) threat versus $100 \%$ (certain) threat (see Figure 7) in humans. This confirms the "classic" stress response dampening effect of alcohol on anxiety ${ }^{38,39}$. Similarly, short term ( 3 day) deprivation of marijuana among heavy daily marijuana users produces selective greater increase in startle potentiation during $20 \%$ (uncertain) threat vs. 100\% (certain) threat in humans (see Figure 8). This result is consistent with current rodent neuroscience evidence that implicates selective "stress neuroadaptation" in brain circuits responsible for anxiety during uncertain threats or other stressors 8.13 .
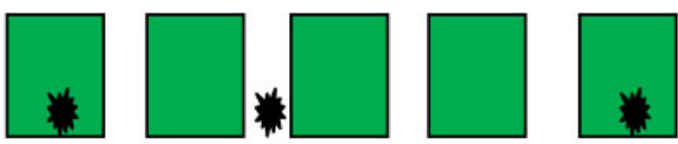

\section{$20 \%$ Shock Probability \\ $100 \%$ Shock Probability}
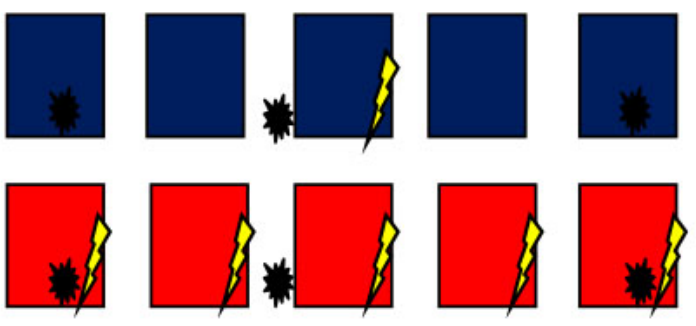

Figure 1. Cues in the Threat Probability Task are divided into two threat of shock (shocks displayed with yellow lightning bolts) probability conditions of $\mathbf{1 0 0} \%$ probability and $\mathbf{2 0} \%$ probability. Shocks occur $4.5 \mathrm{sec}$ into the cue presentation times in threat blocks. Startle potentiation to the threat cues is calculated from startle to sets of no-threat cues. Each cue type is displayed in a different color to facilitate understanding of the current condition. Acoustic startle probes (displayed as black asterisks) are presented 4 sec into the cue presentation times. Acoustic startle probes are also presented at 13 or $15 \mathrm{sec}$ into the ITI periods between the cues. 


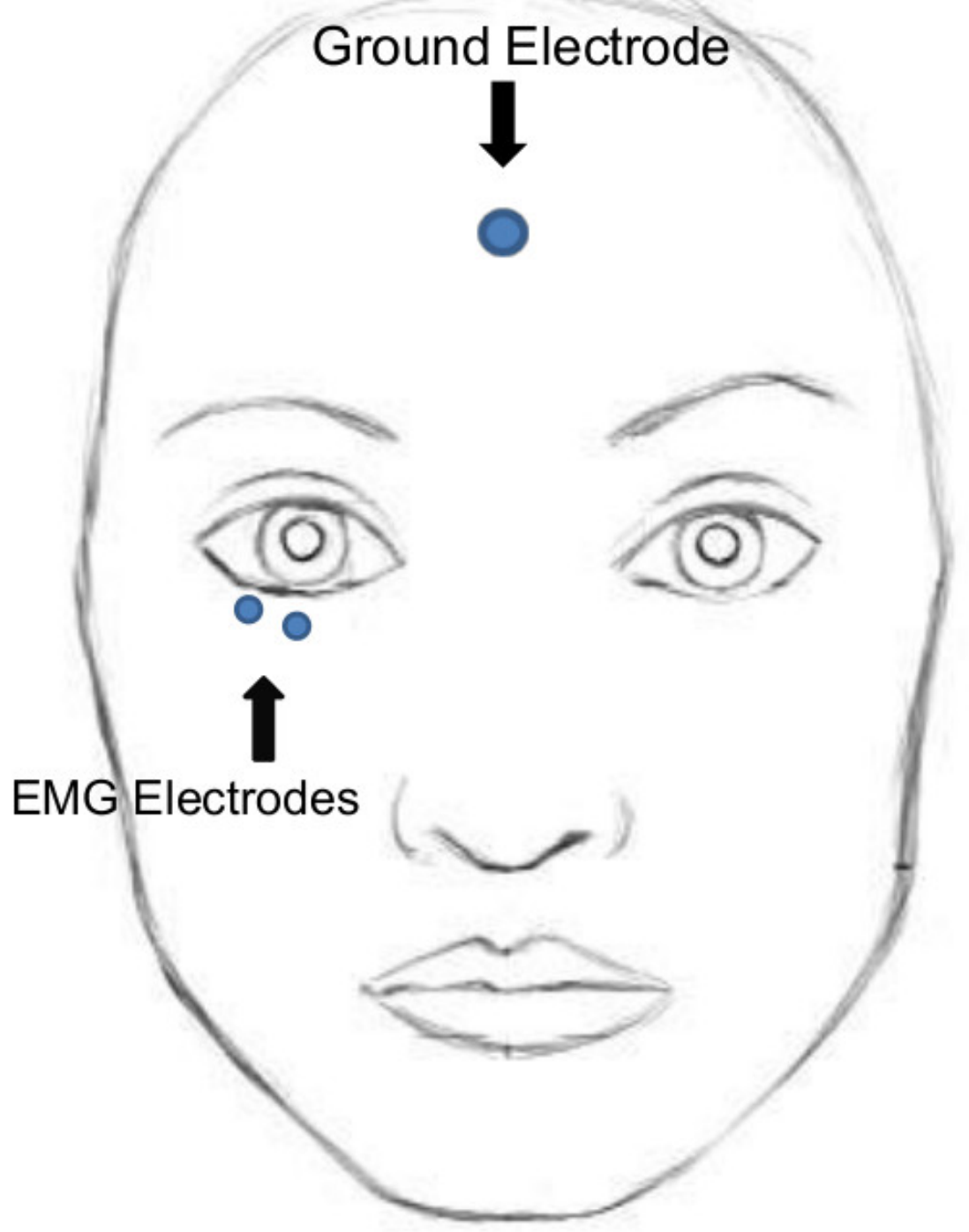

Figure 2. EMG electrode placement. Two small $(4 \mathrm{~mm})$ electro-conductive gel filled EMG Ag-AgCl electrodes are placed over the lower portion of the orbicularis oculi muscle. One large $(8 \mathrm{~mm}) \mathrm{Ag}-\mathrm{AgCl}$ ground electrode is placed on non-focal site such as the participant's forehead. 

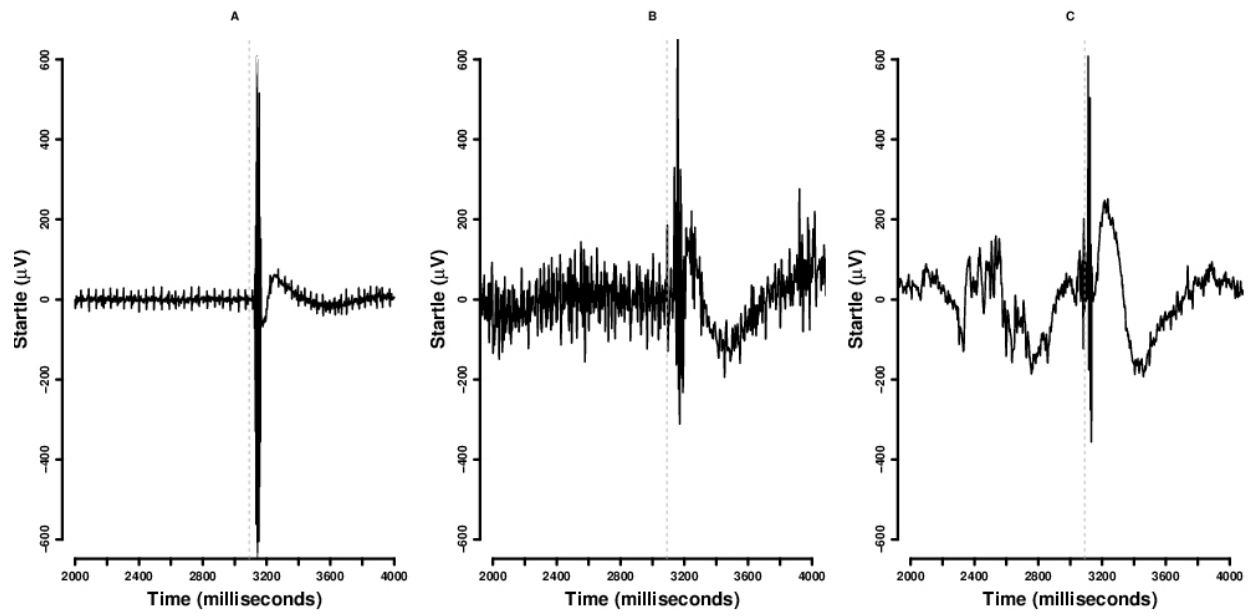

Figure 3. Raw EMG signals. Panel A displays $2 \mathrm{sec}$ of the EMG signal surrounding presentation of a startle probe (vertical gray dotted line). Panel $\mathrm{B}$ displays a similar period of EMG signal that is contaminated by a high degree of electrical noise due to high impedances. Panel $\mathrm{C}$ displays a similar period of EMG signal that is contaminated by movement artifact. Click here to view a larger version of this figure.

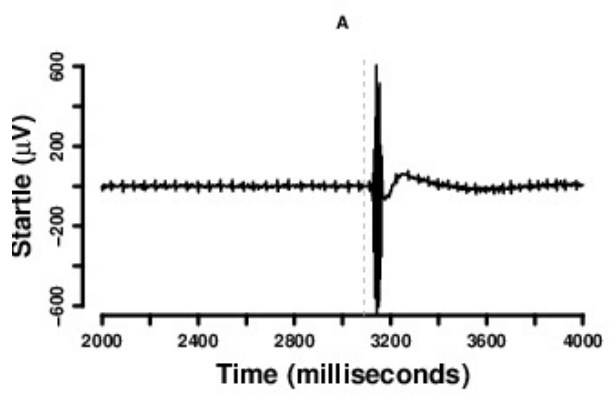

C
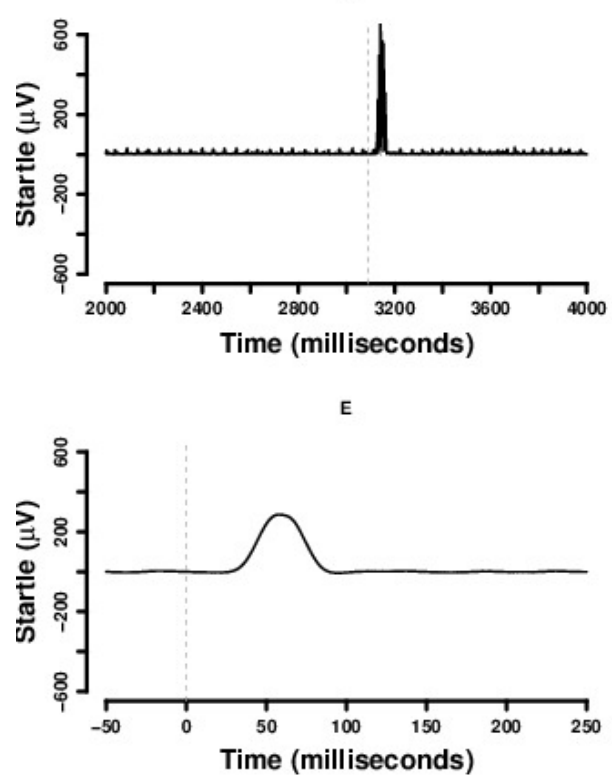

B

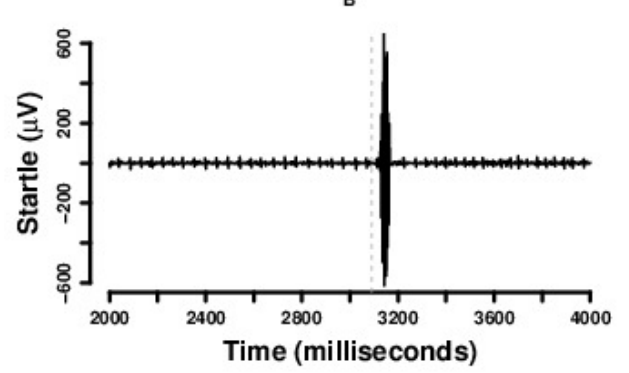

D

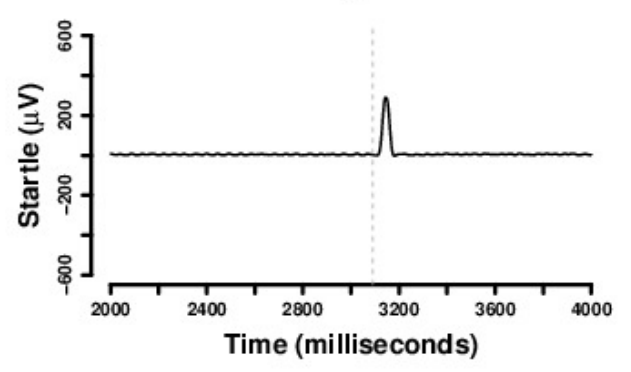

F

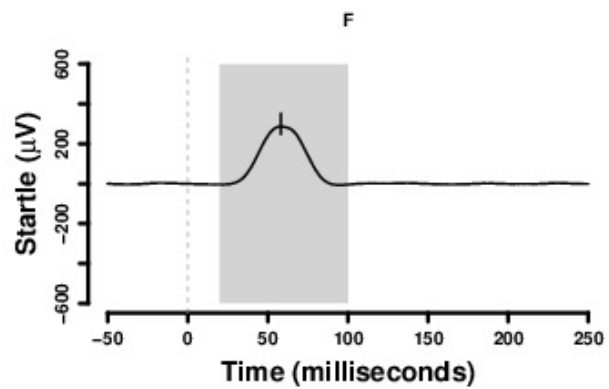

Figure 4. Data Reduction for Startle Response. Panels A-F display a raw EMG signal taken through each processing step described in the protocol. The occurrence of the startle probe is indicated by a vertical gray dotted line) Panel A displays an unprocessed EMG signal. Panel B Displays an EMG signal that has been high pass filtered to remove low frequency artifact. Panel C displays the same EMG signal after rectification. Panel D displays the same EMG signal after it has been smoothed by a low pass filter. Panel E displays the same EMG signal after it has been epoched and baseline corrected. Panel F displays the same EMG signal as panel $\mathrm{E}$ with the gray band representing the $20 \mathrm{msec}$ to $100 \mathrm{msec}$ scoring window for peak startle response (marked with a vertical hatch mark). Click here to view a larger version of this figure. 


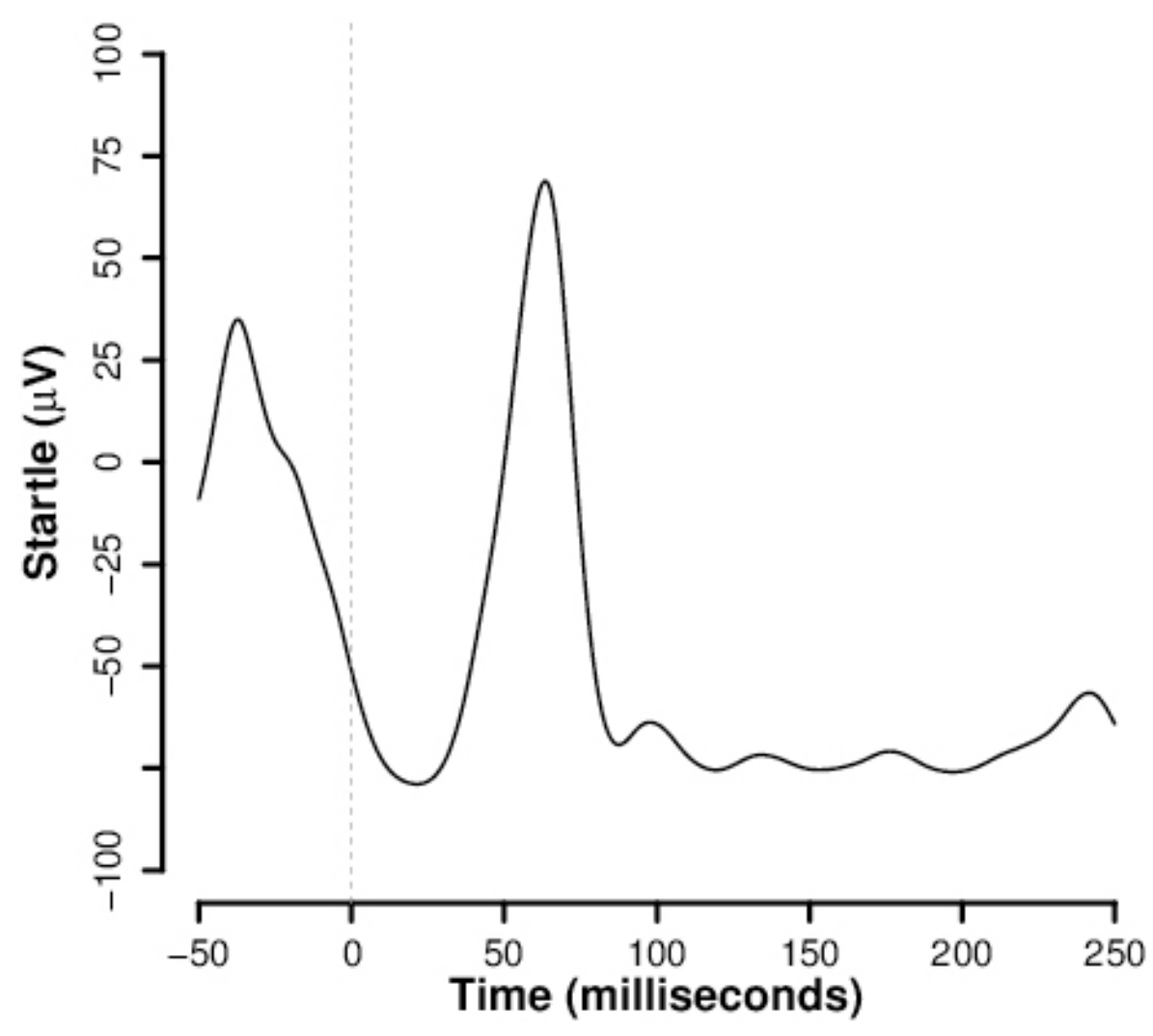

Figure 5. Excessive artifact in pre-probe baseline. A fully processed EMG signal with excessive pre-probe baseline activity. In this case, the participant moved or blinked immediately before the acoustic startle probe presentation (vertical gray dotted line). Click here to view a larger version of this figure.
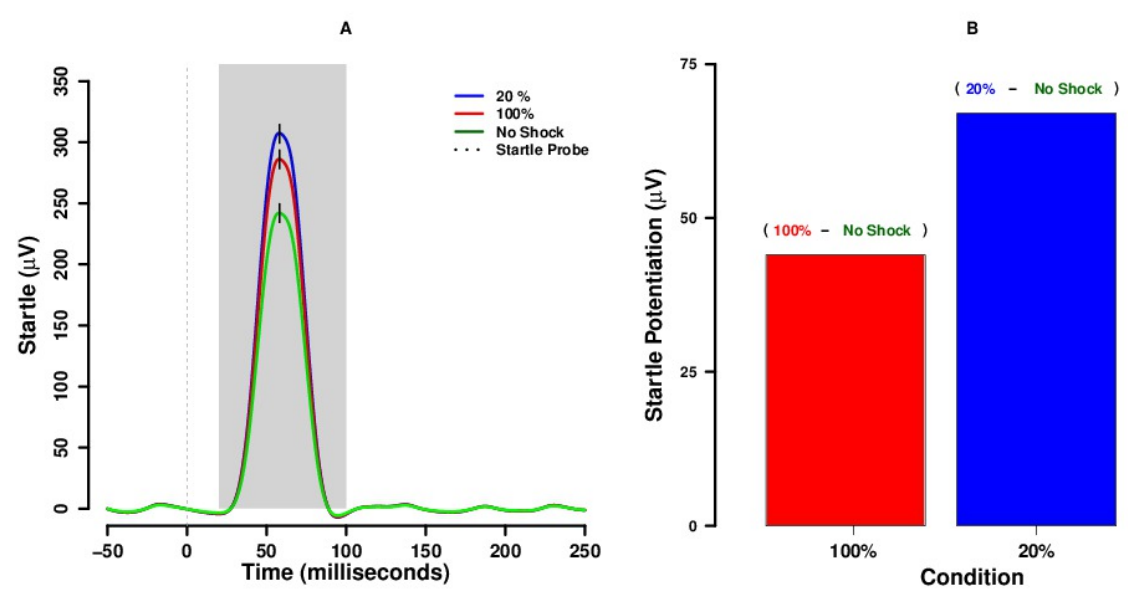

Figure 6. Trial level startle and mean startle potentiation by condition. Panel A shows one fully processed individual trial from each of the three conditions $(20 \%$ shock [in blue], $100 \%$ shock [in red], No Shock [in green]) superimposed on one another. Presentation of the startle probe is indicated by a vertical gray dotted line. The gray band represents the $20 \mathrm{msec}$ to $100 \mathrm{msec}$ scoring window for peak startle response. Peak scored startle response is indicated by a vertical hatch line. Panel B shows the average startle potentiation after subtracting the mean scored response across the 6 individual trials in the No-Shock condition from the mean scored response across the 8 individual trials for each shock condition. Mean startle potentiation for the $100 \%$ shock and $20 \%$ shock conditions are displayed in red and blue, respectively. Click here to view a larger version of this figure. 


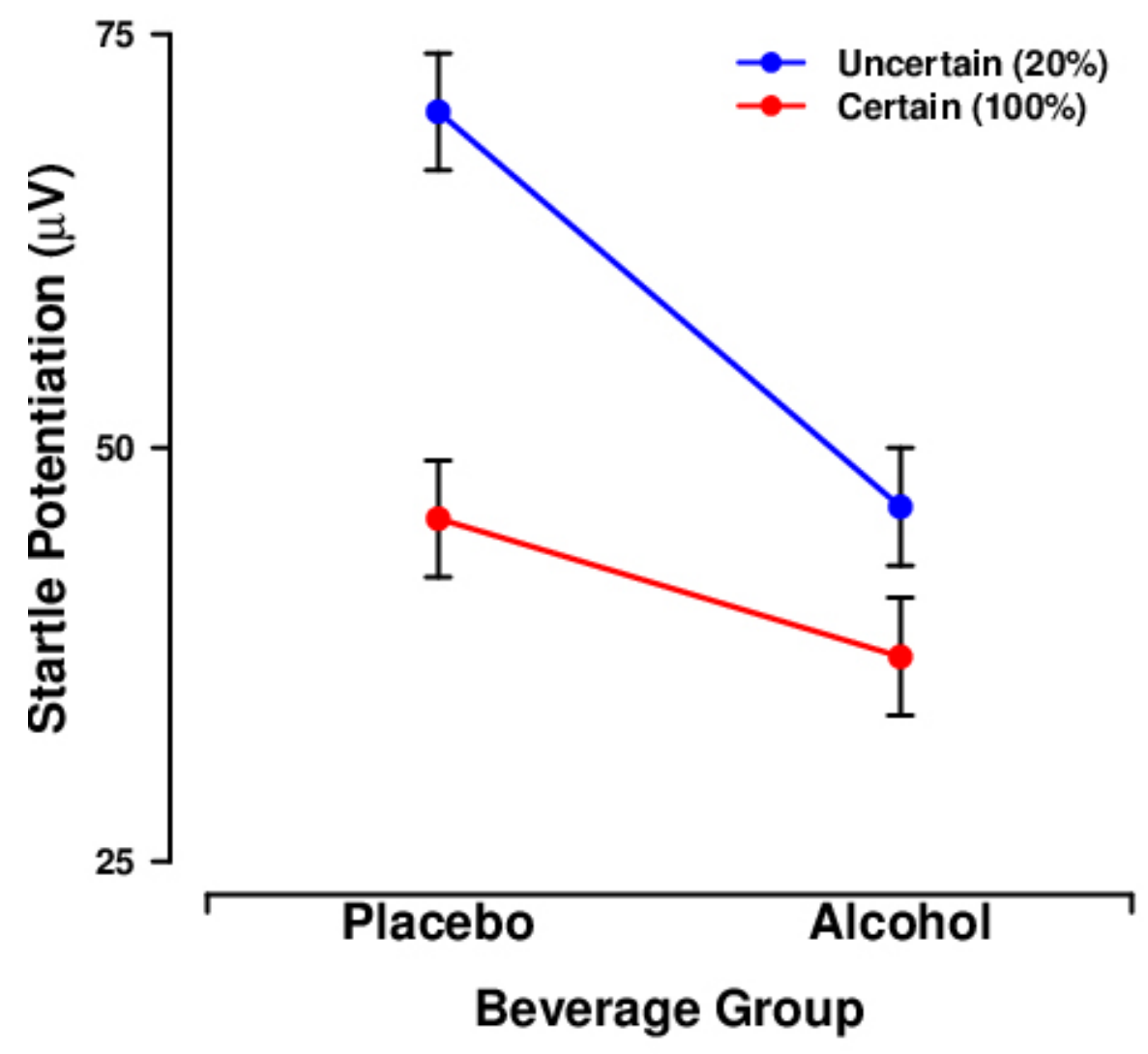

Figure 7. Startle potentiation by Beverage group and threat probability. Mean startle potentiation for the $20 \%$ shock condition is displayed with the blue line. Mean startle potentiation for the $100 \%$ shock condition is displayed with the red line. Standard error of the mean from point estimates of predicted startle potentiation in the General Linear Model are displayed with error bars ${ }^{6}$. Click here to view a larger version of this figure. 


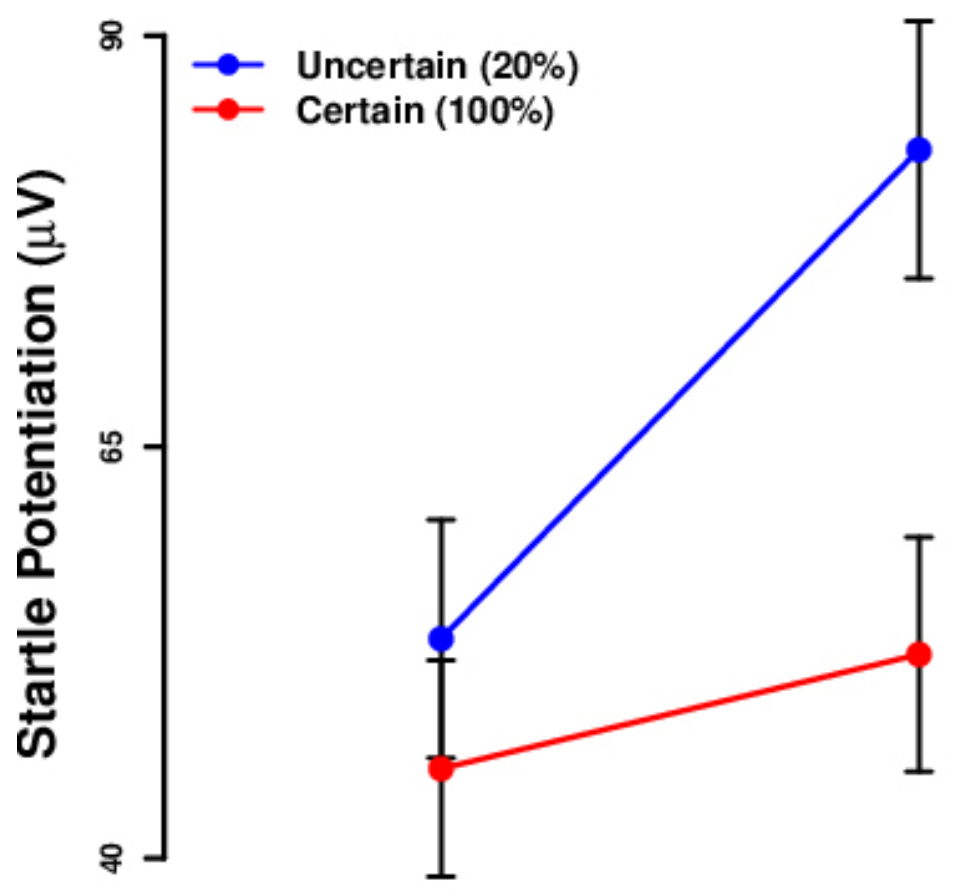

\section{Non-deprived Deprived \\ Marijuana Group}

Figure 8. Startle potentiation by Marijuana group and threat probability. Mean startle potentiation for the $20 \%$ shock condition is displayed with the blue line. Mean startle potentiation for the $100 \%$ shock condition is displayed with the red line. Standard error of the mean from point estimates of predicted startle potentiation in the General Linear Model are displayed with error bars ${ }^{29}$. Click here to view a larger version of this figure.

Supplemental Figure 1. Circuit diagram for the custom shock box used in John Curtin's Addiction Research Laboratory.

Supplemental Table 1. A fully counterbalanced series of trials for the Threat Probability Task. Multiple orders should be used across participants to reduce order effects.

\section{Discussion}

The Threat Probability Task can be used to study the expression of anxiety and fear by assessing startle potentiation to low probability (uncertain) and high probability (certain) threat of electric shock. The primary dependent measure and threat contingencies used in this task can be used with rodents, non-human primates and humans, thus, providing an excellent translational tool for studying the expression of negative affect ${ }^{13,18,40}$. Startle potentiation to threat of electric shock has clear connections to defensive system activation, is resistant to volitional control, and has well defined neurobiological substrates. This is in contrast to self-report measures of affect that may be unduly influenced by demand characteristics and are distal to known neurobiology. The Threat Probability Task offers the precision to parse distinct forms of negative affect in contrasts to other common psychophysiological methods like the Emotional Picture Viewing task. The Threat Probability Task is straightforward to implement and easy to analyze in contrast to other more costly and technical methods such as fMRI.

The Threat Probability Task is one example of a broader class of threat tasks that manipulate threat uncertainty using cued electric shock as the threat and startle potentiation as the dependent measure. As such, there is considerable flexibility in cue-shock contingencies and shock characteristics that can support a program of research with strong conceptual replications. For example, the Threat Probability Task is a derivative of the previously validated No-Shock, Predictable Shock, Unpredictable shock task ${ }^{21}$. The NPU task manipulates uncertainty regarding both IF (shock probability) and WHEN (shock timing) shocks will occur. The NPU task has been used to examine drug administration and deprivation effects on negative affective response ${ }^{4,34}$ and etiological mechanisms in mood and anxiety disorders ${ }^{22-24,41-43}$. In other research, Curtin and colleagues have also developed variants of these cued threat tasks that precisely manipulate threat uncertainty about WHEN (shock timing) ${ }^{5,29,44}$; WHERE (administration location on body for shock) ${ }^{25}$; and HOW BAD (shock intensity) ${ }^{7}$. While Curtin et al.'s use of these tasks has thus far focused on the effects of drug administration and withdrawal in healthy participants; all of these tasks could be used to study anxiety and fear responses in patients with anxiety and other mental disorders ${ }^{2,45}$. 
Curtin and colleagues have used all of the aforementioned classes of cued threat tasks in a program of research that has probed the boundary conditions of alcohol's anxiolytic effects on anxiety expressed during uncertain threat, broadly defined. In all of these tasks, alcohol had a significantly greater stress response dampening effect on startle potentiation during uncertain than certain threat. The consistent pattern of results supports the validity of the threat uncertainty construct and the use of this whole class of tasks to manipulate this construct. Animal neuroscience addiction models suggest that neuroadaptation in the response to uncertain threats and other stressors following repeated, chronic drug use provides one important mechanism in the etiology of alcohol and other drug addiction ${ }^{8}$. Research from our laboratory using these cued threat tasks has provided preliminary support for this etiological mechanism in humans ${ }^{29,34,44}$.

Although startle potentiation provides an attractive translational measure of defensive reactivity to aversive events in the Threat Probability Task, cross-validation with other distinct measures of negative affect responding will reduce concerns about alternative explanations that could be specific to this dependent measure. In fact, the Threat Probability Task can easily accommodate other dependent measures. For example, event related potentials, prepulse inhibition of startle, and behavioral responses can be examined in the Threat Probability Task to allow researchers to probe differences in attentional function during anxiety versus fear ${ }^{15,17,46}$. Researchers interested in anxiety and fear acquisition, rather than expression, can modify the instructions and stimulus parameters in the Threat Probability Task to better serve their research questions. As noted earlier, retrospective self-report of anxiety/fear after each set or at the end of the task can be obtained easily ${ }^{7}$. Online measurement of perceived shock likelihood can also be obtained via keyboard press or voice recording to ensure that participants maintain attention and understanding of instructions throughout the task (for an example see ${ }^{47}$ ). Future work with the Threat Probability Task and similar cued threat tasks can combine other methods while using clinical patient populations to increase the external validity of the task and further define anxiety and fear. For example, it is possible to correlate individual differences in the responding during the Threat Probability Task with responding to threats and other stressors in the "real world" that are assessed via ecological momentary assessment (EMA). Furthermore, participant responding during the Threat Probability Task can be used as a surrogate endpoint ${ }^{48-50}$ to study the effects of pharmacological and/or behavioral interventions to treat mood and anxiety disorders and substance dependence disorders.

The Threat Probability Task can also accommodate threats other than electric shock as well as other startle measurement methods. For example, potentiation of the eyeblink startle response has been confirmed in response to aversive air blast directed at the throat ${ }^{51}$ and aversive loud noise ${ }^{52}$. The startle response can be potentiated by darkness in humans, which provides a clear translational bridge to light potentiated startle in rodents (a nocturnal species ${ }^{13}$ ). The eye-blink startle response can also be elicited by probes in other sensory modalities including visual $^{27}$ and tactile ${ }^{53}$. Clearly, measurement of startle potentiation in the Threat Probability Task and related cued threat tasks provides a flexible tool to affective scientists interested in normative and pathological negative affective response.

\section{Disclosures}

The authors declare that they have no competing financial interests.

\section{Acknowledgements}

This research was supported by Grants R01AA15384 from the National Institute on Alcohol Abuse and Alcoholism and 5R01DA033809-02 from the National Institute of Drug Abuse to John J. Curtin.

\section{References}

1. Barlow, D. H. Unraveling the mysteries of anxiety and its disorders from the perspective of emotion theory. The American psychologist. $\mathbf{5 5}$ (11), 1247-1263, doi:10.1037/0003-066X.55.11.1247 (2000)

2. Boswell, J. F., Thompson-Hollands, J., Farchione, T. J., \& Barlow, D. H. Intolerance of uncertainty: A common factor in the treatment of emotional disorders. Journal of Clinical Psychology. 69 (6), 630-645, doi:10.1002/jclp.21965 (2013).

3. Grillon, C. Models and mechanisms of anxiety: evidence from startle studies. Psychopharmacology. 199 (3), 421-437, doi:10.1007/ s00213-007-1019-1 (2008).

4. Moberg, C. A., \& Curtin, J. J. Alcohol selectively reduces anxiety but not fear: startle response during unpredictable vs. predictable threat. Journal of Abnormal Psychology. 118 (2), 335-347, doi:10.1037/a0015636 (2009).

5. Hefner, K. R., Moberg, C. A., Hachiya, L. Y., \& Curtin, J. J. Alcohol stress response dampening during imminent versus distal, uncertain threat. Journal of abnormal psychology. 122 (3), 756-769, doi:10.1037/a0033407 (2013).

6. Hefner, K. R., \& Curtin, J. J. Alcohol stress response dampening: Selective reduction of anxiety in the face of uncertain threat. Journal of Psychopharmacology (Oxford, England). 26 (2), 232-244, doi:10.1177/0269881111416691 (2012).

7. Bradford, D. E., Shapiro, B. L., \& Curtin, J. J. How bad could it be? Alcohol dampens stress responses to threat of uncertain intensity. Psychological science. 24 (12), 2541-2549, doi:10.1177/0956797613499923 (2013).

8. Koob, G. F., \& Volkow, N. D. Neurocircuitry of addiction. Neuropsychopharmacology Reviews. 35 (1), 217-238, doi:10.1038/npp.2009.110 (2010).

9. Mauss, I. B., \& Robinson, M. D. Measures of emotion: A review. Cognition \& emotion. 23 (2), 209-237, doi:10.1080/02699930802204677 (2009).

10. Lang, P. J., Bradley, M. M., \& Cuthbert, B. N. Emotion, attention, and the startle reflex. Psychological Review. 97 (3), $377-395$, doi:10.1037/0033-295X.97.3.377 (1990).

11. Lang, P. J. The emotion probe. Studies of motivation and attention. The American psychologist. 50 (5), $372-385$ (1995).

12. Vaidyanathan, U., Patrick, C. J., \& Cuthbert, B. N. Linking dimensional models of internalizing psychopathology to neurobiological systems: Affect-modulated startle as an indicator of fear and distress disorders and affiliated traits. Psychological bulletin. 135 (6), 909-942, doi:10.1037/a0017222 (2009).

13. Davis, M., Walker, D. L., Miles, L., \& Grillon, C. Phasic vs sustained fear in rats and humans: Role of the extended amygdala in fear vs anxiety. Neuropsychopharmacology Reviews. 35, 105-135, doi:doi:10.1037/npp.2009 (2010). 
14. Blanchard, R. J., \& Blanchard, D. C. Attack and defense in rodents as ethoexperimental models for the study of emotion. Progress in NeuroPsychopharmacology \& Biological Psychiatry. 13, S3-S14 (1989).

15. Cornwell, B. R., Echiverri, A. M., Covington, M. F., \& Grillon, C. Modality-specific attention under imminent but not remote threat of shock: Evidence from differential prepulse inhibition of startle. Psychological Science. 19 (6), 615- 622, doi:10.1111/j.1467-9280.2008.02131.X (2008).

16. Fanselow, M. S., \& Lester, L. S. A functional behavioristic approach to aversively motivated behavior: predatory imminence as a determinant of the topography of defensive behavior. Evolution and Learning. , 185-212 (1988).

17. Mobbs, D., Petrovic, P., et al. When fear is near: Threat imminence elicits prefrontal-periaqueductal gray shifts in humans. Science. $\mathbf{3 1 7}$ (5841), 1079 -1083, doi:10.1126/science.1144298 (2007).

18. Walker, D., \& Davis, M. Role of the extended amygdala in short-duration versus sustained fear: A tribute to Dr. Lennart Heimer. Brain Structure and Function. 213 (1-2), 29-42, doi:10.1007/s00429-008-0183-3 (2008).

19. Davis, M. Neural systems involved in fear and anxiety measured with fear-potentiated startle. American Psychologist. 61 (8), 741-756, doi:10.1037/0003-066X.61.8.741 (2006).

20. Alvarez, R. P., Chen, G., Bodurka, J., Kaplan, R., \& Grillon, C. Phasic and sustained fear in humans elicits distinct patterns of brain activity. Neurolmage. 55 (1), 389-400, doi:10.1016/j.neuroimage.2010.11.057 (2011).

21. Schmitz, A., \& Grillon, C. Assessing fear and anxiety in humans using the threat of predictable and unpredictable aversive events (the NPUthreat test). Nature Protocols. 7 (3), 527-532, doi:10.1038/nprot.2012.001 (2012).

22. Grillon, C., Lissek, S., Rabin, S., McDowell, D., Dvir, S., \& Pine, D. S. Increased anxiety during anticipation of unpredictable but not predictable aversive stimuli as a psychophysiologic marker of panic disorder. American Journal of Psychiatry. 165 (7), 898-904, doi:10.1176/ appi.ajp.2007.07101581 (2008).

23. Grillon, C., Pine, D. S., Lissek, S., Rabin, S., Bonne, O., \& Vythilingam, M. Increased anxiety during anticipation of unpredictable aversive stimuli in posttraumatic stress disorder but not in generalized anxiety disorder. Biological Psychiatry. 66 (1), 47-53, doi:10.1016/ j.biopsych.2008.12.028 (2009).

24. Grillon, C., Chavis, C., Covington, M. F., \& Pine, D. S. Two-week treatment with the selective serotonin reuptake inhibitor citalopram reduces contextual anxiety but not cued fear in healthy volunteers: A fear-potentiated startle study. Neuropsychopharmacology. 34 (4), 964-971, doi:10.1038/npp.2008.141 (2009).

25. Bradford, D.E., Moberg, C.A., Starr, M.J., Motschman, C.A., Korhumel, R.A., \& Curtin, J.J. Alcohol induced stress neuroadaptation: Cross sectional evidence from startle potentiation and ERPs in healthy drinkers and abstinent alcoholics during uncertain threat. Society for Psychophysiological Research, Abstracts for the Fifty-Third Annual Meeting, Firenze Fiera Congress \& Exhibition Center, Florence, Italy, October 2-6, $2013 \mathrm{http}: / /$ onlinelibrary.wiley.com/doi/10.1111/psyp.12111/full (2013).

26. Shankman, S. A., Robison-Andrew, E. J., Nelson, B. D., Altman, S. E., \& Campbell, M. L. Effects of predictability of shock timing and intensity on aversive responses. International Journal of Psychophysiology: Official Journal of the International Organization of Psychophysiology. $\mathbf{8 0}$ (2), 112-118, doi:10.1016/j.jpsycho.2011.02.008 (2011).

27. Blumenthal, T. D., Cuthbert, B. N., Filion, D. L., Hackley, S., Lipp, O. V., \& van Boxtel, A. Committee report: Guidelines for human startle eyeblink electromyographic studies. Psychophysiology. 42 (1), 1-15, doi:10.1111/j.1469-8986.2005.00271.x (2005).

28. Valsamis, B., \& Schmid, S. Habituation and prepulse inhibition of acoustic startle in rodents. Journal of visualized experiments: JoVE. (55), e3446, doi:10.3791/3446 (2011).

29. Gloria, R. Uncovering a potential biological marker for marijuana withdrawal: Startle potentiation to threat. University of Wisconsin-Madison. 70 p. (2011).

30. Curtin, J. J., Lozano, D., \& Allen, J. B. The psychophysiology laboratory. Oxford University Press: New York, (2007).

31. Lane, S. T., Franklin, J. C., \& Curran, P. J. Clarifying the nature of startle habituation using latent curve modeling. International journal of psychophysiology: official journal of the International Organization of Psychophysiology. 88 (1), 55-63, doi:10.1016/j.ijpsycho.2013.01.010 (2013).

32. Bradford, D. E., Kaye, J. T., \& Curtin, J. J. Not just noise: individual differences in general startle reactivity predict startle response to uncertain and certain threat. Psychophysiology., 51(5), 407-411. doi:10.1111/psyp.12193 (2014).

33. Curtin, J. J., Patrick, C. J., Lang, A. R., Cacioppo, J. T., \& Birbaumer, N. Alcohol affects emotion through cognition. Psychological Science. 12 (6), 527-531, doi:10.1111/1467-9280.00397 (2001).

34. Hogle, J. M., Kaye, J. T., \& Curtin, J. J. Nicotine withdrawal increases threat-induced anxiety but not fear: Neuroadaptation in human addiction. Biological Psychiatry. 68 (8), 687-688, doi:10.1016/j.biopsych.2010.06.003 (2010).

35. Hogle, J. M., \& Curtin, J. J. Sex differences in negative affective response during nicotine withdrawal. Psychophysiology. 43 (4), 344-356, doi:10.1111/j.1469-2006.00406.x (2006).

36. Delorme, A., \& Makeig, S. EEGLAB: an open source toolbox for analysis of single-trial EEG dynamics including independent component analysis. Journal of Neuroscience Methods. 134 (1), 9-21, doi:10.1016/j.jneumeth.2003.10.009 (2004).

37. Statistics Toolbox. Natick, Massachusetts, United States: The Mathworks, Inc. (2013).

38. Levenson, R., Sher, K., Grossman, L., Newman, J., \& Newlin, D. Alcohol and stress response dampening: Pharmacological effects, expectancy, and tension reduction. Journal of Abnormal Psychology. 89 (4), 528-538, doi:10.1037/0021-843X.89.4.528 (1980).

39. Sher, K. J. Stress response dampening. Psychological Theories of Drinking and Alcoholism. $227-271$ (1987).

40. Davis, M., Antoniadis, E., Amaral, D., \& Winslow, J. Acoustic startle reflex in rhesus monkeys: A review. Reviews in the Neurosciences. 19, 171-185, doi:10.1515/REVNEURO.2008.19.2-3.171 (2008).

41. Grillon, C., Baas, J. P., Lissek, S., Smith, K., \& Milstein, J. Anxious responses to predictable and unpredictable aversive events. Behavioral Neuroscience. 118 (5), 916-924, doi:10.1037/0735-7044.118.5.916 (2004).

42. Grillon, C., \& Baas, J. M. A review of the modulation of the startle reflex by affective states and its application in psychiatry. Clinical Neurophysiology. 144 (9), 1557-1579., doi:10.1016/S1388-2457(03)00202-5 (2003).

43. Shankman, S. A., Nelson, B. D., et al. A psychophysiological investigation of threat and reward sensitivity in individuals with panic disorder and/or major depressive disorder. Journal of abnormal psychology. 122 (2), 322-338, doi:10.1037/a0030747 (2013).

44. Moberg, C. A., \& Curtin, J. J. Stressing the importance of anxiety in alcoholism. Alcoholism: Clinical and Experimental Research. 36, 60A, doi:10.1111/j.1530-0277.2012.01803.x (2012).

45. McTeague, L. M., \& Lang, P. J. The anxiety spectrum and the reflex physiology of defense: from circumscribed fear to broad distress. Depression and anxiety. 29 (4), 264-281, doi:10.1002/da.21891 (2012). 
46. Mobbs, D., Marchant, J. L., et al. From Threat to Fear: The Neural Organization of Defensive Fear Systems in Humans. The Journal of Neuroscience. 29 (39), 12236 -12243, doi:10.1523/JNEUROSCI.2378-09.2009 (2009).

47. Lissek, S., Bradford, D. E., et al. Neural substrates of classically conditioned fear-generalization in humans: a parametric fMRI study. Social cognitive and affective neuroscience. , doi:10.1093/scan/nst096 (2013).

48. Insel, T. Next-generation treatments for mental disorders. Science translational medicine. 4 (155), 155ps19, doi:10.1126/ scitransImed.3004873 (2012).

49. Baker, T. B., Mermelstein, R., et al. New methods for tobacco dependence treatment research. Annals of Behavioral Medicine: A Publication of the Society of Behavioral Medicine. 41 (2), 192-207, doi:10.1007/s12160-010-9252-y (2011).

50. Lerman, C., LeSage, M. G., et al. Translational research in medication development for nicotine dependence. Nature Reviews. Drug Discovery. 6 (9), 746-762, doi:10.1038/nrd2361 (2007).

51. Schmitz, A., Merikangas, K., Swendsen, H., Cui, L., Heaton, L., \& Grillon, C. Measuring anxious responses to predictable and unpredictable threat in children and adolescents. Journal of experimental child psychology. 110 (2), 159-170, doi:10.1016/j.jecp.2011.02.014 (2011).

52. Miller, M. W., Curtin, J. J., \& Patrick, C. J. A startle probe methodology for investigating the effects of active avoidance on negative emotional reactivity. Biological Psychology. 50 (3), 235-257, doi:doi:10.1016/S0301-0511(99)00011-3 (1999)

53. Hawk, L. W., \& Cook, E. W., 3rd Affective modulation of tactile startle. Psychophysiology. 34 (1), 23-31, doi:10.1111/ j.1469-8986.1997.tb02412.x (1997). 